\title{
Polycystic Ovarian Syndrome (PCOS) in Obese Metabolic Phenotypes
}

\section{IJCRR}

Section: Healthcare

Sci. Journal Impact

Factor: 6.1 (2018)

ICV: 90.90 (2018)

(c) (i) (3)

Copyright@IJCRR

\section{Neema Acharya ${ }^{1}$, Sourya Acharya ${ }^{2}$, Samarth Shukla ${ }^{3}$, Ketav Joshi ${ }^{4}$, Uplabdh Gopal ${ }^{5}$}

\begin{abstract}
'Professor and Head, Department of Obstetrics and Gynaecology, Jawaharlal Nehru Medical College, Datta Meghe lnstitute of Medical Sciences, Sawangi (Deemed to be University), Maharashtra, India; ${ }^{2}$ Professor, Department of Medicine, Jawaharlal Nehru Medical College,

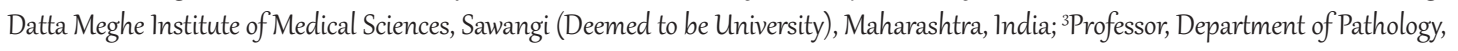
Jawaharlal Nehru Medical College, Datta Meghe Institute of Medical Sciences, Sawangi (Deemed to be University), Maharashtra, India; 4]unior Resident, Department of Obstetrics and Gynaecology, Jawaharlal Nehru Medical College, Datta Meghe Institute of Medical Sciences, Sawangi (Deemed to be University), Maharashtra, India; ${ }^{5}$ Undergraduate student, Department of Obstetrics and Gynaecology, Jawaharlal Nehru Medical College, Datta Meghe Institute of Medical Sciences, Sawangi (Deemed to be University), Maharashtra, India.
\end{abstract}

\section{ABSTRACT}

Introduction: Obesity can be divided into metabolic syndrome (MetS) or metabolically unhealthy obesity (MUO) and metabolically healthy obesity $(\mathrm{MHO})$. In the current scenario, with researches nearing a definition for $\mathrm{MHO}$, it becomes crucial to establish the risks of associated comorbidities of obesity in its subtypes, namely MHO and MetS. With PCOS being generally associated with obesity and an increased risk of cardiovascular diseases, research must be done to determine its relation with $\mathrm{MHO}$ and MetS.

Objectives: Therefore, the purpose of this study is to investigate and compare the key anthropometric and metabolic frameworks of women having PCOS in obese phenotypes, namely, MHO and MetS/MUO in the reproductive age group.

Results: The clinical attributes and metabolic aspects in women having polycystic ovarian syndrome with $\mathrm{MHO}$ will be significantly different than those found in PCOS with MetS/MUO in the reproductive age group.

Conclusion: The clinical and metabolic aspects of women with MHO as study group will be different in PCO women with or without MetS/MUO and may have more risk of development of the cardiovascular disease.

Key Words: Obsesity, Metabolic syndrome, Polycystic ovarian syndrome, Metabolically healthy obesity

\section{INTRODUCTION}

Polycystic ovarian syndrome (PCOS), also known as the Stein-Leventhal syndrome, is a common, yet complex heterogeneous endocrine disorder that affects females in the reproductive age group and is characterised by elevated levels of androgens, menstrual irregularities and associated risks of type 2 diabetes, obesity and coronary artery disease. ${ }^{1,2}$ It is a salient common cause of anovulatory fertility. ${ }^{1}$ The exact prevalence of PCOS has not been defined precisely with estimates for women in the reproductive age ranging from 5-10\%. ${ }^{3,4}$ With revised criteria of Rotterdam (2003), the prevalence of PCOS is expected to rise to $10 \% .{ }^{5,6}$

PCOS usually has a peri-pubertal onset; so, it is a disorder of significant concern, making it all the more important for estimating its effect on the population of our country, and the associated risks, given the paucity of data from India on the topic. Current literature supports the fact that PCOS predisposes the individual to premature cardiovascular disease and subsequent risk for coronary artery disease. ${ }^{7}$ Obesity is a medical state with an excess of body fat contributing to adverse health effects. It is a preventable yet leading cause of death with a rising prevalence worldwide making it a public health issue of utmost importance, especially in adults and children with $39 \%$ of the adult population being overweight and $13 \%$ being obese in the year $2016 .{ }^{8}$ Obesity also increases the likelihood of certain conditions such as cardiovascular disease.

Obesity can be stratified as metabolic syndrome (MetS) or metabolically unhealthy obesity (MUO) and metabolically

\section{Corresponding Author:}

Dr. Neema Acharya, Professor and Head, Department of Obstetrics and Gynaecology, Jawaharlal Nehru Medical College, Datta Meghe Institute of Medical Sciences, (Deemed to be University), Sawangi, Maharashtra, India; Contact: 9326692511; Email: neemasacharya@ gmail.com

ISSN: 2231-2196 (Print)

Received: 26.08 .2020
ISSN: 0975-5241 (Online)

Revised: 24.09 .2020
Accepted: 02.11 .2020

Published: 30.11 .2020 
healthy obesity (MHO). Metabolically healthy obesity is a unique subset that is usually characterised by obesity but without any of its complications. Though there is no universally accepted criteria for defining MHO, with a substantial proportion of obese individuals falling under it, the National Cholesterol Education Program's Adult Treatment Panel

(NCEP-ATP) III criteria to define metabolic syndrome is employed to classify MHO individuals as those satisfying only 2 or less defined parameters. ${ }^{9,10}$ Identification of MHO is an important aspect for studying mechanisms of fat accumulation that contribute to obesity-related CVD risk factors. ${ }^{11,12}$ However, the prognostic value of MHO is highly debatable with seemingly healthy, yet obese individuals presenting with cardiovascular diseases (CVD), osteoarthritis and poor body image. ${ }^{11,12}$ Though MHO individuals are at a decreased risk in comparison to MUO individuals, still, they have an increased risk of progressing to MetS. ${ }^{13,14}$ Studies on comorbidities associated with subtypes of obesity, hence become the need of the hour to assess whether MHO individuals are healthy. ${ }^{10}$

With an increasing prevalence of obesity in most industrialised and developing nations including India, there is evidence suggesting a genetic cause of obesity in the development of PCOS, insulin resistance and infertility. Hence, active intervention becomes a necessity to combat this blight. ${ }^{2}$ In the current scenario, with researches nearing a definition for $\mathrm{MHO}$, it becomes crucial to establish the risks of associated comorbidities of obesity in its subtypes, namely Provide full form is generally associated with obesity and an increased risk of CVD, research must be done to determine its relation with MHO and MetS. Therefore, the purpose of this study is to investigate and compare the key anthropometric and metabolic frameworks of women having PCOS in obese phenotypes, namely, MHO and MetS/MUO in the reproductive age group. Our objective is to ascertain and compare metabolic aspects and clinical attributes in women having polycystic ovarian syndrome with MHO vs. those with MetS/MUO in the reproductive age group.

\section{MATERIALS AND METHODS}

Study Type: Cross-sectional, clinical study with a comparison group

Study Design: The present study will be undertaken on patients in the in-patient department and those attending the out-patient department of Obstetrics and Gynaecology in a rural tertiary care hospital in central India, with a known diagnosis of polycystic ovarian syndrome (PCOS) associated with obesity.

Duration: This study is expected to go long for one year

Sample Size: Data from at least 50 obese patients attending the IPD/OPD with a diagnosis of PCOS. the study will be conducted after due clearance from the Institutional Ethics Committee (IEC). Informed consent will be obtained from the participants before evaluation. The following guidelines and definitions will be used to diagnose and include the study participants:

A. PCOS will be defined according to the revised Rotterdam Criteria.

B. Obesity in this study will be defined as per the body mass index (BMI) categories for Asian Indians as per consensus guidelines. ${ }^{15}$

C. Metabolic syndrome (MetS) will be defined as per the Modified National cholesterol education programme adult treatment panel III (NCEP ATP III) criteria 9 .

D. Most studies suggest the definition of MHO (body mass index $(B M I) \geq 30 \mathrm{~kg} / \mathrm{m}^{2}$ ) to be obesity without the presence of metabolic diseases such as type 2 diabetes, dyslipidaemia or hypertension. ${ }^{16}$

Inclusion criteria: Obese female patients (with a BMI $\geq$ 25.0) ${ }^{15}$ in the reproductive age group with a known diagnosis of PCOS

Exclusion criteria: Patients already receiving treatment for PCOS

- Healthy or overweight patients (with a BMI $\leq 23.0)^{15,16}$

- Known malignancies, pregnancy or other related endocrine disorders

\section{Assessment Criteria}

The selected patients will be evaluated on certain parameters such as anthropometrical measures, blood pressure, biochemical and special investigations performed, as described under the following subheadings:

\section{Biochemical Investigations}

Estimation of plasma glucose levels: Fasting plasma glucose will be estimated by the GOD /POD method by the machine- Robotnik Semi-Automatic Chemical Analyser.

Estimation of serum triglyceride: Serum triglycerides will be estimated using a LIQUID

STABLE GPO (glycerol phosphate oxidase) method.

Estimation of serum HDL: Direct Enzymatic

\section{Special Investigations}

Trans-abdominal Ultrasonography will be conducted to confirm a diagnosis ${ }^{5}$ by the machine- Arietta S70 Ultrasound Colour Doppler, Hitachi Medical Systems (Aloka Company).

\section{DATA ANALYSIS}

It will be performed with the help of Statistical Package SPSS (version 25.0), IBM Corporation and Excel 2013, 
Microsoft Corporation. Multiple logistic regression analysis will be performed to identify and examine the objective predictors of PCOS and their impact. The chi-square test and student T-test will also be employed to test for the strength of association between variables and probability among the phenotypes.

\section{EXPECTED OUTCOMES}

The metabolic aspects and clinical attributes in women having Polycystic ovarian syndrome with $\mathrm{MHO}$ will be significantly different from those which will be found in women with MetS/MUO in the reproductive age.

\section{DISCUSSION}

The study will categorise the participants into MHO and MetS phenotypes and compare the two groups in terms of metabolic parameters and clinical characteristics. It will help to determine if a significant difference exists between these two phenotypes having PCOS. Hence, it will be helpful in the management of these women in terms of specific targeted therapy, long term lifestyle modifications and screening for risks of comorbidities associated with these phenotypes. ${ }^{16,17}$ Furthermore, if a substantial amount of MHO population suffers from PCOS, it makes them further vulnerable for future cardio-vascular perils, suggesting MHO is an increased risk state and PCOS additionally contributes towards the established risks of cardiovascular morbidities.

\section{CONCLUSION}

The clinical and metabolic aspects of women with $\mathrm{MHO}$ as study group will be different in PCO women with or without MetS/MUO and hence have a higher risk of development of cardiovascular disease in future life.

\section{ACKNOWLEDGMENT}

Authors acknowledge the immense help received from the scholars whose articles are cited and included in references to this manuscript. The authors are also grateful to authors / editors / publishers of all those articles, journals, and books from which the literature for this article has been reviewed and discussed.

\section{Conflict of Interest: Nil}

Source of Funding: Nil

\section{REFERENCES}

1. Teede H, Deeks A, Moran L. Polycystic ovary syndrome: a complex condition with psychological, reproductive and metabolic manifestations that impacts on health across the lifespan. BMC Med 2010;8(1):41.

2. Allahbadia GN, Merchant R. Polycystic ovary syndrome in the Indian Subcontinent. Semi Reprod Med 2008;26(01):22-34.

3. Knochenhauer ES, Key TJ, Kahsar-Miller M, Waggoner W, Boots LR, Azziz R. Prevalence of the polycystic ovary syndrome in unselected black and white women of the southeastern United States: A prospective study. J Clin Endocrinol Metab 1998;83(9):3078-82.

4. Azziz R, Woods KS, Reyna R, Key TJ, Knochenhauer ES, Yildiz BO. The prevalence and features of Polycystic Ovary Syndrome in an unselected population. J Clin Endocrinol Metab 2004;89(6):2745-9.

5. Rotterdam ESHRE/ASRM-Sponsored PCOS Consensus Workshop Group. Revised 2003 consensus on diagnostic criteria and long-term health risks related to polycystic ovary syndrome. Fertil Steril 2004;81(1):19-25.

6. Nidhi R, Padmalatha V, Nagarathna R, Amritanshu R. Prevalence of the polycystic ovarian syndrome in Indian adolescents. J Pediatr Adolesc Gynecol 2011;24(4):223-7.

7. Dokras A. Cardiovascular disease risk in women with PCOS. Steroids 2013;78(8):773-6.

8. World Health Organization. Obesity and overweight. Fact sheet N 311. http://www. who. int/mediacentre/factsheets/fs311/en/. 2012.

9. Expert Panel on Detection, and Treatment of High Blood Cholesterol in Adults. Executive Summary of the third report of the National Cholesterol Education Program (NCEP) expert panel on detection, evaluation, and treatment of high blood cholesterol in adults (Adult Treatment Panel III). JAMA 2001;285:2486-97.

10. Plourde G. Metabolically Healthy Obese (MHO) in Adults and Adolescents: Where We Are. J Gerontol Geriatr Med 2018;4(2):555634.

11. Plourde G, Karelis AD. Current issues in the identification and treatment of metabolically healthy but obese individuals. Nutr Metab Cardiovasc Dis 2014;24(5):455-9.

12. Jung $\mathrm{CH}$, Lee WJ, Song K. Metabolically healthy obesity: a friend or foe? Korean J Intern Med 2017;32(4):611-21.

13. Acharya S, Shukla S. Metabolic Healthy Obesity- A Paradoxical fallacy? J Clin Diagn Res 2018;12(10):07-10.

14. Hinnouho GM, Czernichow S, Dugravot A, Nabi H, Brunner EJ, Kivimaki M, et al. Metabolically healthy obesity and the risk of cardiovascular disease and type 2 diabetes: The Whitehall II cohort study. Eur Heart J 2015;36(9):551-9.

15. Misra A, Chowbey P, Makkar BM, Vikram NK, Wasir JS, Chadha $\mathrm{D}$, et al. Consensus statement for diagnosis of obesity, abdominal obesity and the metabolic syndrome for Asian Indians and recommendations for physical activity, medical and surgical management. J Assoc Physicians India. 2009;57:163-70.

16. Bobbioni-Harsch E, Pataky Z, Makoundou V, Laville M, Disse $\mathrm{E}$, Anderwald $\mathrm{C}$, et al. From metabolic normality to cardiometabolic risk factors in subjects with obesity. Obesity (Silver Spring) 2012;20(10):2063-69.

17. Sagar V, Wanjari A, Kumar S, Munshi AP. Echocardiographic Assessment in Various Obesity Phenotypes. Int J Pharma Res 2019; 11(2): 1804-7. 